\title{
IS THE DEFORMATION SPACE OF COMPLETE AFFINE STRUCTURES ON THE 2-TORUS SMOOTH?
}

\author{
OLIVER BAUES AND WILLIAM M. GOLDMAN
}

Dedicated to Alberto Verjovsky on his sixtieth birthday

\begin{abstract}
Periods of parallel exterior forms define natural coordinates on the deformation space of complete affine structures on the two-torus. These coordinates define a differentiable structure on this deformation space, under which it is diffeomorphic to $\mathbb{R}^{2}$. The action of the mapping class group of $T^{2}$ is equivalent in these coordinates with the standard linear action of $\operatorname{SL}(2, \mathbb{Z})$ on $\mathbb{R}^{2}$.
\end{abstract}

\section{INTRODUCTION}

Conformal structures on the 2-torus $T^{2}$ (elliptic curves) are classified by a moduli space, which is the quotient of the upper half-plane $\mathrm{H}^{2}$ by the action of the modular group $\mathrm{SL}(2, \mathbb{Z})$ by linear fractional transformations. In other words, equivalence classes of elliptic curves correspond to orbits of $\mathrm{SL}(2, \mathbb{Z})$ on $\mathrm{H}^{2}$. Since $\mathrm{H}^{2} / \mathrm{SL}(2, \mathbb{Z})$ is not a smooth manifold, it is often easier to study properties of elliptic curves in terms of the action of $\mathrm{SL}(2, \mathbb{Z})$ on the smooth manifold $\mathrm{H}^{2}$.

This note concerns the analogous question for complete affine structures on $T^{2}$. A complete affine structure on a manifold $M$ is a representation of $M$ as the quotient of affine space $\mathbb{A}^{n}$ by a discrete group of affine transformations. Equivalence classes of such structures on $T^{2}$ identify with the orbits of $\mathrm{SL}(2, \mathbb{Z})$ on $\mathbb{R}^{2}$ for the standard linear action of $\mathrm{SL}(2, \mathbb{Z})$ on $\mathbb{R}^{2}$. However, unlike the action on $\mathrm{H}^{2}$, this action is not proper and the quotient space is even more badly behaved. In particular it fails to be Hausdorff and enjoys a nonstandard Borel structure.

Date: January 12, 2004.

Goldman gratefully acknowledges partial support from National Science Foundation grant DMS-0103889. This research was performed at the Séminaire SudRhodanien at the Centre international de recherches en mathématiques in Luminy in November 2001, and the workshop "Discrete Groups and Geometric Structures, with Applications" held at the Katolieke Universiteit Leuven Campus Kortrijk, in Kortrijk, Belgium in March 2002. We are grateful for the hospitality afforded by these two meetings. 
The coordinates in $\mathrm{H}^{2}$, parametrizing elliptic curves, are defined by the periods of holomorphic 1-forms. Analogously, we use periods of parallel 1-forms to define coordinates on the deformation space of complete affine structures on $T^{2}$. These coordinates define a natural differentiable structure on this deformation space, under which it is diffeomorphic to $\mathbb{R}^{2}$. (Using a different approach Baues [1] noticed that this deformation space is homeomorphic to $\mathbb{R}^{2}$.)

Whereas every smooth deformation of elliptic curves is induced by a smooth map into $\mathrm{H}^{2}$, the analogous result fails for the deformation space of complete affine structures on $T^{2}$ with respect to the coordinates given by the periods of parallel 1-forms. We provide an example of a smooth one-parameter deformation of an Euclidean 2-torus, in fact an affine fibration, which corresponds to a continuous curve in the deformation space which is not smooth in the origin.

The complete affine structures on $T^{2}$ were classified by Kuiper [18]. In this paper we describe the moduli space of such structures. As is often the case in moduli problems, the moduli space can be made more tractable by introducing extra structure - in this case a marking, and the moduli problem for marked structures is simpler. Equivalence classes of marked structures form a space homeomorphic to the plane $\mathbb{R}^{2}$, which we call the deformation space. The moduli space - consisting of equivalence classes of structures (without marking) — is then the orbit space of $\mathbb{R}^{2}$ by the standard linear action of $\operatorname{SL}(2, \mathbb{Z})$.

That the deformation space of complete affine structures on $T^{2}$ is even Hausdorff is in itself surprising. (The larger deformation space of (not necessarily complete) affine structures on $T^{2}$ is not Hausdorff.)

Moreover, in our setup the group actions defining the moduli problem are not reductive, and hence the usual techniques of geometric invariant theory do not apply. Indeed, the affine structures discussed here all come from affine structures on abelian Lie groups which are invariant under multiplication, and the resulting automorphisms of the affine structure form a unipotent group.

Benzecri [5] (see also Milnor [22]) proved that the only compact orientable surface which admits affine structures is the two-torus $T^{2}$. The classification of affine structures on $T^{2}$ up to affine diffeomorphism, initiated by Kuiper [18], was completed independently by FurnessArrowsmith [9] and Nagano-Yagi [23].

According to Kuiper, the complete affine structures on the 2-torus fall into two types. Quotients of $\mathbb{A}^{2}$ by translations we call Euclidean structures, since they admit compatible Euclidean (or flat Riemannian) metrics. The Levi-Civita connection of a compatible metric coincides 
with the flat connection associated to the affine structure. The Euclidean structures fall into a single affine equivalence class, which corresponds to the origin in $\mathbb{R}^{2}$. The other structures are more exotic, and do not correspond to Levi-Civita connections of a (possibly indefinite) Riemannian metric.

The non-Riemannian structures can be characterized as follows. Let $M$ be a marked non-Riemannian affine 2-torus. There is a parallel area form $\omega_{M}$ which we normalize to have area 1 . Then there exists a vector field $\xi_{M}$, polynomial of degree 1 , such that the covariant derivative $\zeta_{M}=\nabla_{\xi_{M}} \xi_{M}$ is a nonzero parallel vector field. Since parallel vector fields on $M$ are determined up to multiplication by a nonzero scalar, we can normalize $\zeta_{M}$ by requiring that

$$
\omega_{M}\left(\xi_{M}, \zeta_{M}\right)=1
$$

Then the interior product

$$
\eta_{M}:=\iota_{\zeta_{M}}\left(\omega_{M}\right)
$$

is a parallel 1 -form on $M$, uniquely determined by the affine structure of $M$. The cohomology class

$$
\left[\eta_{M}\right] \in H^{1}\left(T^{2} ; \mathbb{R}\right)
$$

determines the equivalence class of the marked affine manifold $M$. As non-Euclidean structures converge to the Euclidean structure, this cohomology class approaches zero, and the period mapping extends to a homeomorphism of the deformation space of all complete marked affine structures on $T^{2}$ with $\mathbb{R}^{2}$.

The case when the periods are rationally related, that is when $\left[\eta_{M}\right]$ lies in $\mathbb{R} \cdot H^{1}\left(T^{2} ; \mathbb{Z}\right)$, is particularly notable. In this case the parallel vector field $\zeta_{M}$ has closed trajectories, and the homology class of a trajectory is Poincaré dual to $\left[\eta_{M}\right]$. Equivalently, $\eta_{M}$ integrates to an affine fibration $M \longrightarrow S^{1}$, where $S^{1}$ is given a complete affine structure.

For every complete affine structure on $T^{2}$ there is a commutative group operation on $T^{2}$ for which multiplication is affine. Thus every complete affine 2-torus $M$ is a group object in the category of affine manifolds. Such objects correspond to associative algebras, providing an alternative approach to the classification. From this viewpoint, a marked complete affine structure on $T^{2}$ corresponds to a commutative associative nilpotent $\mathbb{R}$-algebra, with a chosen basis corresponding to the marking.

Similarly Matsushima [21] showed that the set of all complex affine structures on a fixed complex torus forms an affine algebraic variety, by 
identifying each structure with the associative algebra which is formed by the holomorphic vector fields of the torus under the covariant differentiation of the affine structure.

As a related fact the deformation spaces of complete affine structures on (also higher dimensional) real tori are homeomorphic to a real algebraic set $[2]$.

In figure 1-9 we show various tilings of the plane which are obtained from affine group actions corresponding to different values of the period class. The Mathematica notebook used for creating these pictures is available from http://ww. math.umd.edu/ res/EGL/AffineT2.nb. An interactive Java applet for investigating these structures can be found at http://www.math.umd.edu/ lidador/Affine/. 


\begin{tabular}{|l|l|l|l|l|l|l|l|l|l|}
\hline & & & & & & & & & \\
\hline & & & & & & & & & \\
\hline & & & & & & & & & \\
\hline & & & & & & & & & \\
\hline & & & & & & & & & \\
\hline & & & & & & & & & \\
\hline & & & & & & & & & \\
\hline & & & & & & & & & \\
\hline & & & & & & & & & \\
\hline & & & & & & & & & \\
\hline
\end{tabular}

Figure 1. Period Class $=(0,0)$

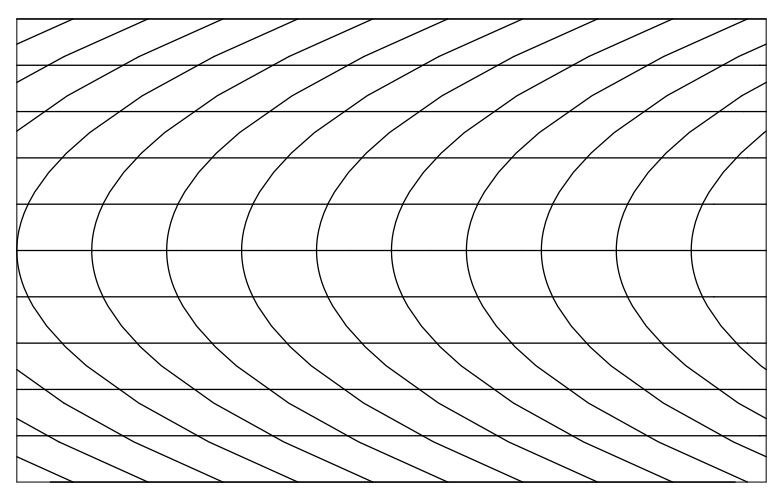

Figure 2. Period Class $=(0.7,0)$

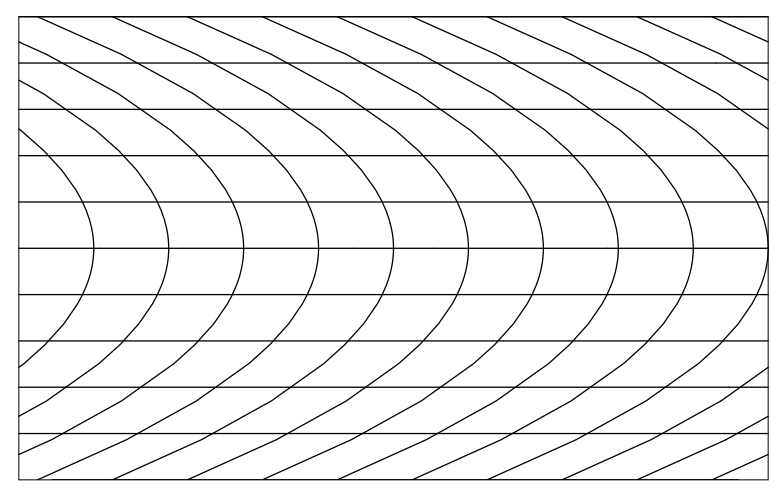

Figure 3. Period Class $=(-0.7,0)$ 


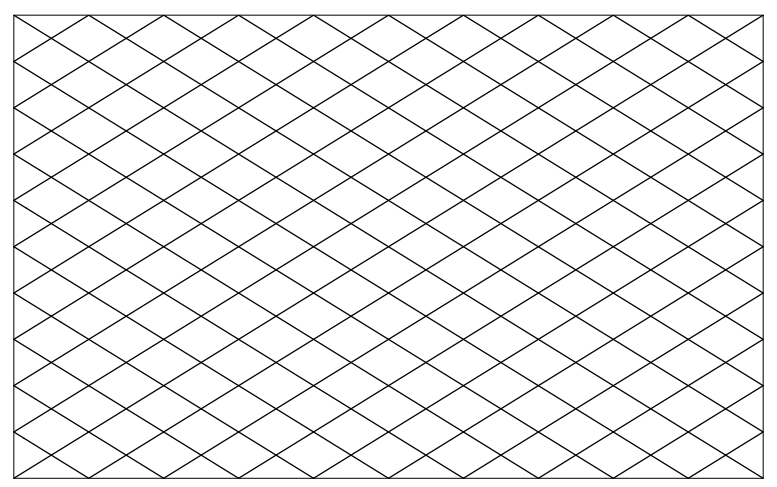

Figure 4. Period Class $=(0,0)$

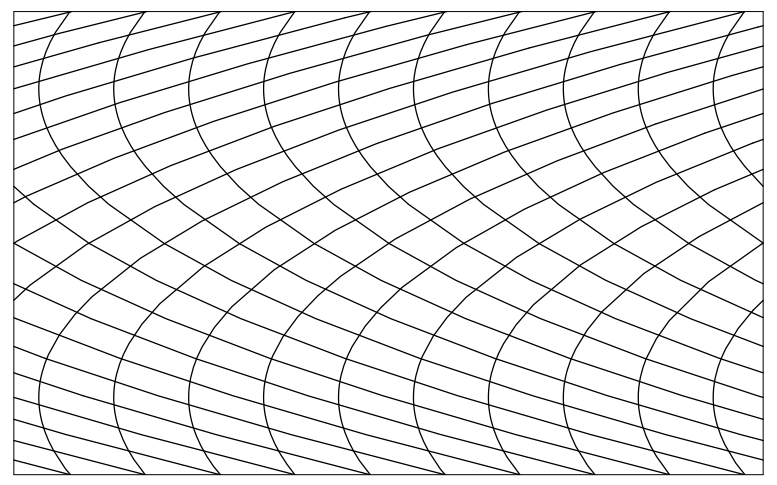

Figure 5. Period Class $=(0.5,0.5)$

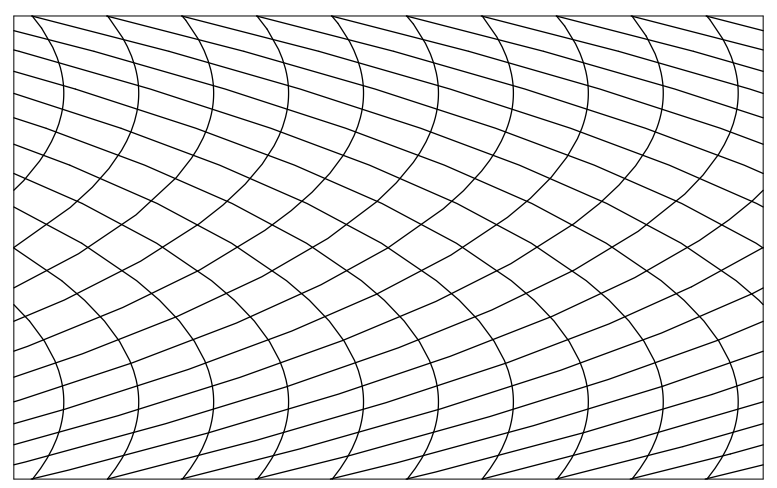

Figure 6. Period Class $=(-0.5,-0.5)$ 


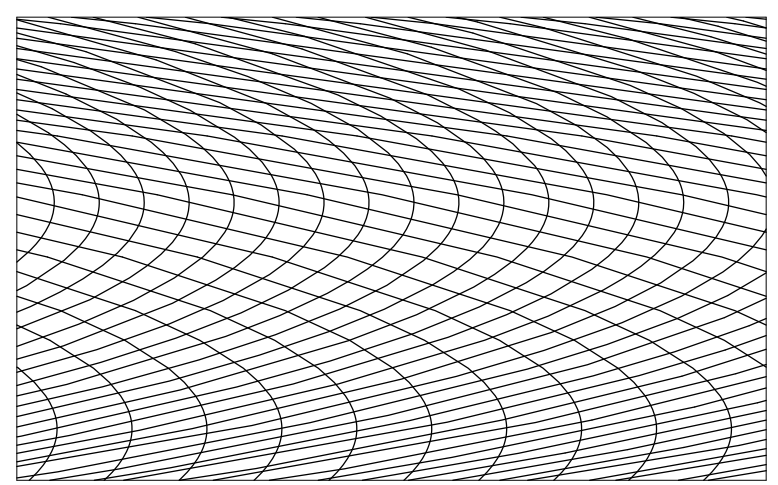

Figure 7. Period Class $=(0.7,1.2)$

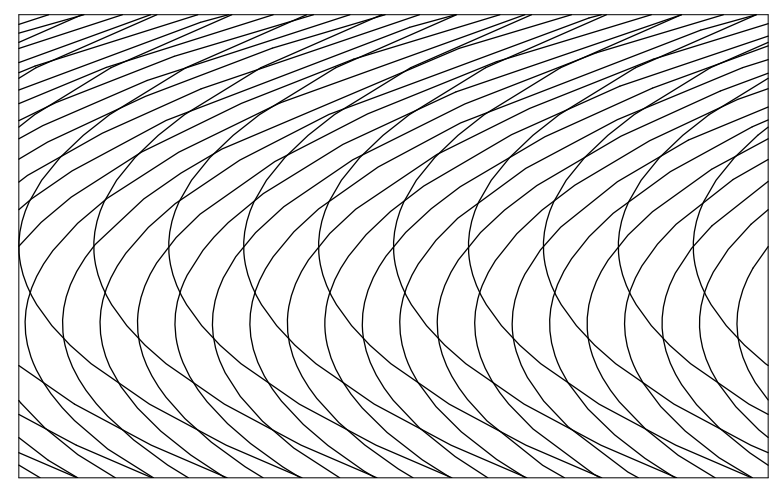

Figure 8. Period Class $=(-1.7,-1.9)$

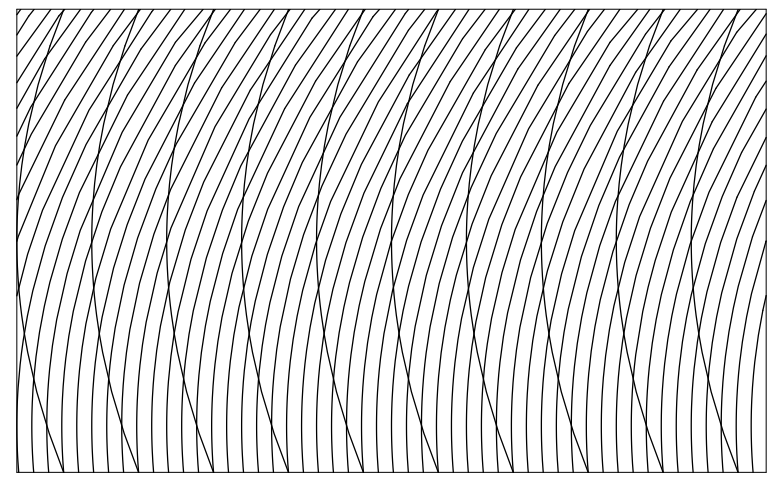

Figure 9. Period Class $=(-0.6,-1.5)$ 


\section{Generalities on affine structures}

Let $\mathbb{A}^{n}$ denote real affine $n$-space with an orientation. Let $\operatorname{Aff}(n)$ denote its group of orientation-preserving affine automorphisms. We use the standard linear representation. $\mathbb{A}^{n}$ is the affine hyperplane $\mathbb{R}^{n} \times\{1\} \subset \mathbb{R}^{n+1}$ and $\operatorname{Aff}(n)$ the subgroup of $\mathrm{GL}(n+1 ; \mathbb{R})$ consisting of matrices

$$
(A, b):=\left[\begin{array}{cc}
A & b \\
0 & 1
\end{array}\right]
$$

where $A \in \mathrm{GL}(n)$ and $b \in \mathbb{R}^{n}$. The affine action is defined by

$$
(v, 1) \stackrel{(A, b)}{\longmapsto}(A v+b, 1) .
$$

An affine structure on an oriented smooth manifold $M$ is a maximal atlas of coordinate charts into $\mathbb{A}^{n}$ such that on overlapping coordinate patches the transformation is locally the restriction of an element of $\operatorname{Aff}(n)$. The manifold $M$ together with an affine structure is called an affine manifold. A local diffeomorphism $N \stackrel{f}{\longrightarrow} M$ between affine manifolds is affine if it is related to affine isomorphisms by the local affine coordinate charts. Given a local diffeomorphism $f$, an affine structure $M$ determines a unique affine structure on $N$ such that $f$ is an affine map. Thus covering spaces, and the universal covering space $\tilde{M} \longrightarrow M$ in particular, of affine manifolds are themselves affine manifolds. We shall assume for convenience that our affine manifolds are path-connected and oriented.

Let $M$ be an affine manifold. The affine coordinate atlas on $M$ globalizes to a developing map

$$
\operatorname{dev}: \tilde{M} \rightarrow \mathbb{A}^{n}
$$

from the universal covering $\tilde{M}$ into $\mathbb{A}^{n}$. This affine map determines the affine structure on $M$, and is uniquely determined (for a given affine structure) by its restriction to a coordinate patch. Conversely, any affine chart on a coordinate patch extends to a unique developing map.

Let $M$ be an affine manifold and $p \in M$. A affine germ at $p$ is an equivalence class of affine diffeomorphisms

$$
U \stackrel{\psi}{\rightarrow} \psi(U) \subset \mathbb{A}^{n}
$$

where $U$ is a neighborhood of $p$ in $M$ and $\psi(U) \subset \mathbb{A}^{n}$ is open. Affine diffeomorphisms $U \stackrel{\psi}{\rightarrow} \mathbb{A}^{n}$ and $U^{\prime} \stackrel{\psi^{\prime}}{\longrightarrow} \mathbb{A}^{n}$ define the same germ if and only if they agree on $U \cap U^{\prime}$. Clearly $\operatorname{Aff}(n)$ acts simply transitively on the set of affine germs at $p \in M$. Since an affine germ determines 
a unique developing map, the developing map of a affine manifold is unique up to composition with an affine transformation $g \in \operatorname{Aff}(n)$.

Deck transformations of $\tilde{M} \rightarrow M$ act by affine automorphisms of $\tilde{M}$ and define the affine holonomy representation $\pi_{1}(M) \stackrel{h}{\longrightarrow} \operatorname{Aff}(n)$ for which dev is equivariant. The developing pair (dev, $h$ ) is unique up to the action of $\operatorname{Aff}(n)$ where $\operatorname{Aff}(n)$ acts by left-composition on dev and by conjugation on $h$.

An affine manifold is complete if its developing map is a diffeomorphism. Equivalently, an affine manifold is complete if it is affinely diffeomorphic to a quotient space of $\mathbb{A}^{n}$ by a discrete group $\Gamma$ of affine transformations acting properly on $\mathbb{A}^{n}$. An affine crystallographic group is a discrete subgroup of $\operatorname{Aff}(n)$ acting properly with compact quotient. The classification of complete affine manifolds up to affine diffeomorphism is equivalent to the classification of affine crystallographic groups up to affine conjugacy.

Our goal is to construct a space whose points represent inequivalent affine structures on a given closed manifold $S$. Unfortunately such spaces are typically non-Hausdorff quotients of singular spaces which are not smooth manifolds. Thus we replace our goal with finding a smooth manifold (or at least a smoothly stratified space) with a group action, whose orbits represent affine diffeomorphism classes of affine structures on $S$.

To compare different affine manifolds, define a marked affine manifold as an orientation-preserving diffeomorphism $S \stackrel{f}{\rightarrow} M$ where $M$ is an oriented affine manifold. The group $\operatorname{Diff}(S)$ of all orientationpreserving diffeomorphisms $S \stackrel{\phi}{\rightarrow} S$ acts by composition with $f$ on the set of affine manifolds which are marked by $S$ :

$$
S \stackrel{\phi}{\rightarrow} S \stackrel{f}{\rightarrow} M
$$

Two affine manifolds $(f, M)$ and $\left(f^{\prime}, M^{\prime}\right)$ marked by $S$ are considered isomorphic if there exists an affine diffeomorphism $\varphi: M \rightarrow M^{\prime}$ such that $\varphi \circ f=f^{\prime}$, they are isotopic if there exists an affine diffeomorphism $\varphi: M \rightarrow M^{\prime}$ such that $\varphi \circ f$ and $f^{\prime}$ are related by a diffeomorphism in the identity component $\operatorname{Diff}_{0}(S)$ of $\operatorname{Diff}(S)$.

Let $x_{0} \in S$ be a basepoint, $\Pi: \tilde{S} \longrightarrow S$ the corresponding universal covering space, and $\pi=\pi_{1}\left(S, x_{0}\right)$ its group of deck transformations. Let $(f, M)$ be an affine manifold marked by $S$. An affine germ $[\psi]$ at $f\left(x_{0}\right)$ determines a developing pair $(\operatorname{dev}, h)$, which is defined on $\tilde{S}$. This development pair depends only on the affine isomorphism class of the pair $((f, M),[\psi])$. 
Let $\operatorname{Def}\left(S, x_{0}\right)$ denote the set of all affine isomorphism classes of pairs $((f, M),[\psi])$. One can alternatively think of $\operatorname{Def}\left(S, x_{0}\right)$ as the set of developing pairs $(\mathrm{dev}, h)$. Since the developing map dev determines the affine holonomy $h$, one can further regard $\operatorname{Def}\left(S, x_{0}\right)$ as the set of developing maps of affine structures on $S$. Affine holonomy defines a map

$$
\operatorname{Def}\left(S, x_{0}\right) \stackrel{\text { hol }}{\longrightarrow} \operatorname{Hom}(\pi, \operatorname{Aff}(n))
$$

which is invariant under the subgroup $\operatorname{Diff}_{0}\left(S, x_{0}\right)$ of $\operatorname{Diff}_{0}(S)$ fixing $x_{0}$, since the elements of $\operatorname{Diff}_{0}\left(S, x_{0}\right)$ induce the identity map on $\pi=$ $\pi_{1}\left(S, x_{0}\right)$.

These spaces are topologized as follows. Give $\operatorname{Def}\left(S, x_{0}\right)$ the $C^{\infty}$ topology of developing maps: a sequence of developing maps converges if and only if it and all of its derivatives converge uniformly on compact subsets. Give $\operatorname{Def}\left(S, x_{0}\right) / \operatorname{Diff}_{0}\left(S, x_{0}\right)$ the quotient topology. If $\pi$ is finitely generated, then $\operatorname{Hom}(\pi, \operatorname{Aff}(n))$ is a real affine algebraic set, and has the classical topology. A sequence $h_{m} \in \operatorname{Hom}(\pi, \operatorname{Aff}(n))$ converges if and only if for all finite subsets $\left\{\alpha_{1}, \ldots, \alpha_{N}\right\} \subset \pi$, and all compact subsets $K \subset \mathbb{A}^{n}$, the restrictions of $h_{m}\left(\alpha_{i}\right)$ to $K$ converge uniformly for $i=1, \ldots, N$.

In these topologies, hol is continuous.

Thurston [29] was the first to realize that hol defines a local homeomorphism

$$
\operatorname{Def}\left(S, x_{0}\right) / \operatorname{Diff}_{0}\left(S, x_{0}\right) \stackrel{\text { Hol }}{\longrightarrow} \operatorname{Hom}(\pi, \operatorname{Aff}(n)),
$$

that is, hol is an open map and two nearby developing maps with identical holonomy are isotopic by a basepoint-preserving isotopy. See $[6,13,15,19]$ for further discussion of this general fact.

In general, the holonomy map $\mathrm{Hol}$ is not injective, even if $S$ is compact. The simplest example occurs when $S=T^{2}$ and the holonomy has cyclic image (covering spaces of Hopf manifolds). See Smillie[26], Sullivan-Thurston [28] and Goldman [11] for more exotic examples.

However, the restriction of $\mathrm{Hol}$ to complete structures is injective and identifies complete structures with their holonomy representations.

Since $\pi$ is discrete, a representation $h \in \operatorname{Hom}(\pi, \operatorname{Aff}(n))$ defines a proper action if for all compact $K_{1}, K_{2} \subset \mathbb{A}^{n}$, the set

$$
\left\{\alpha \in \pi \mid \alpha\left(K_{1}\right) \cap K_{2} \neq \emptyset\right\}
$$

is finite. If furthermore $h$ defines a free action, then $\pi$ is torsionfree. In particular the quotient $\mathbb{A}^{n} / h(\pi)$ is Hausdorff, the quotient map $\mathbb{A}^{n} \longrightarrow$ $\mathbb{A}^{n} / h(\pi)$ is a covering space, and $\mathbb{A}^{n} / h(\pi)$ inherits a smooth structure from $\mathbb{A}^{n}$. 
Let $\operatorname{Hom}_{\mathrm{p}, \mathrm{S}}(\pi, \operatorname{Aff}(n))$ denote the subset of $\operatorname{Hom}(\pi, \operatorname{Aff}(n))$ consisting of proper actions $h$ such that the quotient $\mathbb{A}^{n} / h(\pi)$ is diffeomorphic to $S$. Let $\operatorname{Diff}^{0}\left(S, x_{0}\right)$ denote the subgroup of $\operatorname{Diff}\left(S, x_{0}\right)$ which induces the identity on $\pi_{1}\left(S, x_{0}\right)$.

Proposition 1.1. Let $\operatorname{Def}_{\mathrm{c}}\left(S, x_{0}\right)$ denote the subspace of $\operatorname{Def}\left(S, x_{0}\right)$ corresponding to complete affine structures. Then the restriction

$$
\operatorname{Def}_{\mathrm{c}}\left(S, x_{0}\right) / \operatorname{Diff}^{0}\left(S, x_{0}\right) \stackrel{\text { Hol }}{\longrightarrow} \operatorname{Hom}_{\mathrm{p}, \mathrm{S}}(\pi, \operatorname{Aff}(n))
$$

is a homeomorphism.

We sketch the proof. Let $h \in \operatorname{Hom}_{\mathrm{p}, \mathrm{S}}(\pi, \operatorname{Aff}(n))$. A diffeomorphism $S \longrightarrow \mathbb{A}^{n} / h(\pi)$ defines a complete structure on $S$ with holonomy $h$, showing surjectivity of Hol. If $S \stackrel{f}{\rightarrow} M$ is a marked affine structure on $M$ such that the holonomy $h$ is the holonomy of a marked complete affine structure

$$
S \stackrel{f^{\prime}}{\rightarrow} M^{\prime}=\mathbb{A}^{n} / h(\pi),
$$

then the development map

$$
\tilde{S} \stackrel{\tilde{f}}{\rightarrow} \tilde{M} \stackrel{\operatorname{dev}_{M}}{\longrightarrow} \mathbb{A}^{n}
$$

descends to a local diffeomorphism $S \stackrel{F}{\rightarrow} M^{\prime}$ inducing the isomorphism

$$
\pi_{1}\left(S, x_{0}\right) \longrightarrow \pi_{1}\left(M^{\prime}, f\left(x_{0}\right)\right)=h(\pi)
$$

defined by $h$. Since $S$ is closed, the local diffeomorphism is a covering space and since $\pi_{1}(F)$ is an isomorphism, $F$ is a diffeomorphism. The composition $\left(f^{\prime}\right)^{-1} \circ F \in \operatorname{Diff}(S)$ induces the identity on $\pi_{1}\left(S, x_{0}\right)$. This completes the sketch.

Evidently hol is $\operatorname{Aff}(n)$-equivariant, where $\operatorname{Aff}(n)$ acts by composition of the affine germ on $\operatorname{Def}\left(S, x_{0}\right)$ and by conjugation on $\operatorname{Hom}(\pi, \operatorname{Aff}(n))$. The space $\operatorname{Def}^{\prime}\left(S, x_{0}\right)$ of isomorphism classes of marked affine manifolds $(f, M)$ identifies with the quotient $\operatorname{Def}\left(S, x_{0}\right) / \operatorname{Aff}(n)$, and hol defines a continuous map

$$
\operatorname{Def}^{\prime}\left(S, x_{0}\right) \longrightarrow \operatorname{Hom}(\pi, \operatorname{Aff}(n)) / \operatorname{Aff}(n) .
$$

Note that $\operatorname{Aut}\left(\pi_{1}\left(S, x_{0}\right)\right)$ acts on $\operatorname{Hom}(\pi, \operatorname{Aff}(n))$ by right composition on homomorphisms. Furthermore hol is equivariant with respect to the $\operatorname{Diff}\left(S, x_{0}\right)$-action on $\operatorname{Def}\left(S, x_{0}\right)$ and the action on $\operatorname{Hom}(\pi, \operatorname{Aff}(n))$ defined by the homomorphism

$$
\operatorname{Diff}\left(S, x_{0}\right) \longrightarrow \operatorname{Aut}\left(\pi_{1}\left(S, x_{0}\right)\right) .
$$

The space of isotopy classes of marked complete affine manifolds $(f, M)$ identifies with the quotient $\mathfrak{D}_{c}(S)=\operatorname{Def}_{c}^{\prime}\left(S, x_{0}\right) / \operatorname{Diff}_{0}\left(S, x_{0}\right)$ and is called the deformation space. The $\operatorname{Diff}\left(S, x_{0}\right)$-action on $\mathfrak{D}_{c}(S)$ factors 
through the discrete group $\pi_{0}\left(\operatorname{Diff}\left(S, x_{0}\right)\right)$ and the homeomorphism Hol of Proposition 1.1 induces a surjective map

$$
\begin{aligned}
& \mathfrak{D}_{c}(S) / \pi_{0}\left(\operatorname{Diff}\left(S, x_{0}\right)\right) \longrightarrow \\
&\left(\operatorname{Hom}_{\mathrm{p}, \mathrm{S}}(\pi, \operatorname{Aff}(n)) / \operatorname{Aff}(n)\right) / \operatorname{Aut}\left(\pi_{1}\left(S, x_{0}\right)\right) .
\end{aligned}
$$

The first space is the moduli space, consisting of affine diffeomorphism classes of complete affine manifolds which are diffeomorphic to $S$. In this paper we show that when $S=T^{2}$, then this space identifies naturally with the quotient of $\mathbb{R}^{2}$ by $\operatorname{SL}(2, \mathbb{Z})$.

Example. In general, neither $\operatorname{Def}^{\prime}\left(S, x_{0}\right)$ nor $\operatorname{Hom}(\pi, \operatorname{Aff}(n)) / \operatorname{Aff}(n)$ is Hausdorff. Two-dimensional Hopf manifolds provide counterexamples. Namely the quotients

$$
M_{\epsilon}:=\left(\mathbb{R}^{2}-\{(0,0)\}\right) /\left\{\left[\begin{array}{ll}
2 & \epsilon \\
0 & 2
\end{array}\right]^{n} \mid n \in \mathbb{Z}\right\}
$$

are affine 2-tori. All the structures with $\epsilon \neq 0$ are equivalent, but are not equivalent to $M_{0}$. In particular the equivalence class of $M_{1}$ contains the equivalence class of $M_{0}$ in its closure.

Remarks. The conventions in this paper were chosen to simplify the discussion of our specific example: complete affine structures on $T^{2}$. In general, one may to want to work with markings which are only homeomorphisms or homotopy equivalences, or with manifolds which are not oriented (or nonorientable). We have chosen to work in the oriented differentiable category for convenience, thereby avoiding as many topological technicalities as possible. Similarly one may want to avoid the discussion of base-point preserving diffeomorphisms in discussing the mapping class group (see $\S 2$ ), but in the interest of brevity we have chosen the present approach to avoid the general complications.

Also in the specific simple case $S=T^{2}$ which is treated in this paper, we have that $\operatorname{Hom}_{\mathrm{p}, \mathrm{S}}(\pi, \operatorname{Aff}(n))$ coincides with the subset of $\operatorname{Hom}(\pi, \operatorname{Aff}(n))$ consisting of all proper actions of $\pi$, and the condition on the quotient space is actually superfluous. In fact, if $\pi=\mathbb{Z}^{2}$ then $\mathbb{A}^{2} / h(\pi)$ must be diffeomorphic to the 2-torus from the classification of surfaces. (See [2] for further discussion, and generalization to higher-dimensional examples.) Moreover, since $T^{2}$ is a surface, $\operatorname{Diff}_{0}\left(T^{2}, x_{0}\right)=\operatorname{Diff}^{0}\left(T^{2}, x_{0}\right)$, see $\S 2$. 
Connections. An affine structure is equivalent to a flat torsionfree affine connection. Recall that an affine connection is a covariant differentiation operation on vector fields

$$
\begin{aligned}
\operatorname{Vect}(M) \times \operatorname{Vect}(M) & \stackrel{\nabla}{\longrightarrow} \operatorname{Vect}(M) \\
(X, Y) & \longmapsto \nabla_{X}(Y)
\end{aligned}
$$

which is $\mathbb{R}$-bilinear, and for $f \in C^{\infty}(M)$, satisfies

$$
\nabla_{f X} Y=f \nabla_{X} Y, \quad \nabla_{X}(f Y)=f \nabla_{X} Y+(X f) Y
$$

(where $X f \in C^{\infty}(M)$ denotes the directional derivative of $f$ with respect to $X)$. The connection is torsionfree if and only if

$$
\nabla_{X} Y-\nabla_{Y} X=[X, Y]
$$

and flat if and only if

$$
\nabla_{X} \nabla_{Y}-\nabla_{Y} \nabla_{X}=\nabla_{[X, Y]}
$$

Conversely any such affine connection arises from an atlas of locally affine coordinates as above. (Compare Kobayashi-Nomizu [16].)

Covariant differentiation extends to other tensor fields by enforcing identities such as the following. For example, if $\eta$ is a 1-form, and $X \in \operatorname{Vect}(M)$, then $\nabla_{X}(\eta)$ is the 1-form defined by:

$$
\left(\nabla_{X}(\eta)\right)(Y)=-\eta\left(\nabla_{X} Y\right)
$$

for all $Y \in \operatorname{Vect}(M)$. If $\eta_{1}, \eta$ are 1-forms, then

$$
\nabla_{X}\left(\eta_{1} \wedge \eta_{2}\right)=\nabla_{X}\left(\eta_{1}\right) \wedge \eta_{2}-\eta_{1} \wedge \nabla_{X}\left(\eta_{2}\right)
$$

(and linearity) defines $\nabla_{X} \eta$ for any exterior 2-form $\eta$.

A tensor field $\eta$ is parallel if $\nabla_{X} \eta=0$ for all $Y \in \operatorname{Vect}(M)$. Equivalently, in local affine coordinates the coefficients of $\eta$ are constants. More generally $\eta$ is polynomial of degree $\leq r$ if and only if

$$
\nabla_{X_{1}} \cdots \nabla_{X_{r+1}}(\eta)=0
$$

for all $X_{1}, \ldots, X_{r+1} \in \operatorname{Vect}(M)$.

\section{The TWO-TORUS $T^{2}$}

Let $S$ be the two-torus $T^{2}$, that is the quotient space of $\mathbb{R}^{2}$ by the action of the integer lattice $\mathbb{Z}^{2} \subset \mathbb{R}^{2}$ by translations. The quotient map

$$
\tilde{S}=\mathbb{R}^{2} \longrightarrow \mathbb{R}^{2} / \mathbb{Z}^{2}=T^{2}
$$

is a universal covering space, thereby providing global Euclidean coordinates for $\tilde{S}$. Let the image of the origin $0 \in \mathbb{R}^{2}$ be the basepoint $x_{0} \in T^{2}$. 
A developing map for $T^{2}$ is thus a local diffeomorphism

$$
\mathbb{R}^{2} \stackrel{\operatorname{dev}}{\longrightarrow} \mathbb{A}^{2}
$$

which is equivariant with respect to the translation action of $\pi \cong \mathbb{Z}^{2}$ on $\mathbb{R}^{2}$, and an affine action defined by a homomorphism $\rho: \pi \rightarrow \operatorname{Aff}(2)$ :

$$
\mathrm{p}+\left[\begin{array}{l}
m_{1} \\
m_{2}
\end{array}\right] \stackrel{\text { dev }}{\longmapsto}\left(\gamma_{1}\right)^{m_{1}}\left(\gamma_{2}\right)^{m_{2}} \mathrm{p}
$$

where $\gamma_{i}=\rho\left(\alpha_{i}\right)$ for $i=1,2$ and

$$
\alpha_{1}=\left[\begin{array}{l}
1 \\
0
\end{array}\right], \quad \alpha_{2}=\left[\begin{array}{l}
0 \\
1
\end{array}\right]
$$

generate the fundamental group $\pi \cong \mathbb{Z}^{2}$.

The modular group. The mapping class group of $T^{2}$ is particularly tractable. Every orientation-preserving diffeomorphism $T^{2} \longrightarrow T^{2}$ is isotopic to a basepoint-preserving diffeomorphism, and every isotopy between basepoint-preserving diffeomorphisms can be deformed to a basepoint-preserving isotopy. Furthermore the natural map

$$
\operatorname{Diff}\left(S, x_{0}\right) \longrightarrow \operatorname{Aut}\left(\pi_{1}\left(S, x_{0}\right)\right)
$$

corresponds to an isomorphism

$$
\pi_{0}\left(\operatorname{Diff}\left(T^{2}\right)\right) \stackrel{\cong}{A} u t\left(\pi_{1}\left(T^{2}, x_{0}\right)\right) \cong \operatorname{SL}(2, \mathbb{Z}) .
$$

See $\S 6.4$ of Stillwell [27] for discussion.

$\mathrm{SL}(2, \mathbb{Z})$ is explicitly realized by linear diffeomorphisms of $T^{2}$ as follows. A matrix $A \in \mathrm{SL}(2, \mathbb{Z})$ defines a linear automorphism of $\mathbb{R}^{2}$ which normalizes the action of integer translations $\mathbb{Z}^{2}$. Thus $A$ induces a diffeomorphism of the quotient $T^{2}=\mathbb{R}^{2} / \mathbb{Z}^{2}$, which preserves the basepoint $x_{0}$. Thus the extension

$$
1 \longrightarrow \operatorname{Diff}_{0}\left(T^{2}\right) \longrightarrow \operatorname{Diff}\left(T^{2}\right) \longrightarrow \pi_{0}\left(\operatorname{Diff}\left(T^{2}\right)\right) \cong \mathrm{SL}(2, \mathbb{Z}) \longrightarrow 1
$$

is split.

\section{Complete affine structures on $T^{2}$}

Euclidean structures. Since translations of $\mathbb{R}^{2}$ are affine transformations, $\mathbb{Z}^{2}$ acts as an affine crystallographic group and $T^{2}$ inherits a complete affine structure from the structure of $\mathbb{R}^{2}$ (its universal covering) as a vector space. The generators $\gamma_{1}, \gamma_{2}$ are translations by linearly independent vectors in $\mathbb{R}^{2}$ spanning a lattice in $\mathbb{R}^{2}$. Furthermore any two lattices in $\mathbb{R}^{2}$ are equivalent by a linear automorphism of $\mathbb{R}^{2}$, so different lattices in $\mathbb{R}^{2}$ define affinely diffeomorphic structures. This affine 
structure is called the Euclidean structure since it supports more refined geometric structures modelled on Euclidean geometry. (Of course, most linear automorphisms will not preserve this more refined metric structure.)

The affine equivalence class of the Euclidean structure will be the origin in the deformation space. The mapping class group $\operatorname{SL}(2, \mathbb{Z})$ fixes this affine equivalence class.

Simply transitive affine actions. However, $T^{2}$ carries many other non-equivalent affine structures. These structures are not subordinate to Riemannian metrics, so we call them non-Riemannian. All the structures arise from simply transitive unipotent affine actions, and we briefly review the general theory.

By Smillie [26] or Fried-Goldman-Hirsch [8], any proper affine action of an abelian (or, more generally, nilpotent) group is unipotent. That is, the linear parts are unipotent linear transformations. Equivalently the corresponding elements of $\mathrm{GL}(n+1)$ are unipotent. Furthermore a subgroup consisting of unipotent elements is conjugate to the upper triangular unipotent subgroup of $\mathrm{GL}(n+1)$.

If $\Gamma \subset \operatorname{Aff}(n)$ is an affine crystallographic group which is nilpotent, then there exists a unique simply connected unipotent subgroup $G$ of $\operatorname{Aff}(n)$ containing $\Gamma$ which acts simply transitively on $\mathbb{A}^{n}$. Indeed, $G$ is the algebraic hull of $\Gamma$ in $\operatorname{Aff}(n)$, that is, the Zariski closure of $\Gamma$ in $\operatorname{Aff}(n)$.

Let $\mathfrak{g}$ denote the Lie algebra of $G$. The exponential map $\mathfrak{g} \stackrel{\exp }{\longrightarrow} G$ is a diffeomorphism.

For any $p \in \mathbb{A}^{n}$, the composition

$$
\mathfrak{g} \stackrel{\exp }{\longrightarrow} G \stackrel{\mathrm{ev}_{p}}{\longrightarrow} \mathbb{A}^{n}
$$

is a polynomial diffeomorphism of the vector space $\mathfrak{g}$ with the affine space $\mathbb{A}^{n}$, where.

$$
\begin{aligned}
G \stackrel{\mathrm{ev}_{p}}{\longrightarrow} \mathbb{A}^{n} \\
g \longmapsto g(p)
\end{aligned}
$$

denotes evaluation at $p \in \mathbb{A}^{n}$. Hence any two unipotent affine crystallographic actions of a group $\Gamma$ are conjugate by a polynomial diffeomorphism of $\mathbb{A}^{n}$ ([7], Theorem 1.21).

Furthermore this implies that the de Rham cohomology of the quotient $M=\mathbb{A}^{n} / \Gamma$ is computable from the cohomology of polynomial exterior differential forms on $M([14])$. Since $\Gamma$-invariant polynomial tensor fields on $\mathbb{A}^{n}$ are invariant under the algebraic hull $G$, every tensor at the origin $p \in \mathbb{A}^{n}$ extends uniquely to a $\Gamma$-invariant polynomial 
tensor field on $\mathbb{A}^{n}$. Conversely, any two $\Gamma$-invariant polynomial tensor fields which agree at $p$ are equal. Thus the space of polynomial vector fields on $M$ identifies with the tangent space $T_{p} \mathbb{A}^{n}=\mathbb{R}^{n}$, the space of polynomial $1-$ forms on $M$ identifies with the dual space $T_{p}^{*} \mathbb{A}^{n}=\left(\mathbb{R}^{n}\right)^{*}$. Since a unipotent subgroup of $\operatorname{Aff}(n)$ preserves volume, the top-dimensional cohomology is represented by a parallel volume form.

Here is an explicit construction for $n=2$. Every unipotent subgroup is conjugate to a subgroup of the multiplicatively closed affine subspace $1+\mathfrak{N}_{3} \subset \mathrm{GL}(3)$ where

$$
\mathfrak{N}_{3}:=\left\{\left[\begin{array}{lll}
0 & * & * \\
0 & 0 & * \\
0 & 0 & 0
\end{array}\right]\right\}
$$

is the subalgebra of strictly upper triangular matrices. The algebra $\mathfrak{N}_{3}$ satisfies $\mathfrak{N}_{3}{ }^{3}=0$, and thus the exponential and logarithm maps are given by:

$$
\begin{aligned}
1+\mathfrak{N}_{3} & \stackrel{\log }{\longrightarrow} \mathfrak{N}_{3} \\
\gamma & \longmapsto(\gamma-1)-\frac{1}{2}(\gamma-1)^{2}
\end{aligned}
$$

and

$$
\begin{aligned}
\mathfrak{N}_{3} & \stackrel{\exp }{\longrightarrow} 1+\mathfrak{N}_{3} \\
a & \longmapsto \\
& \longmapsto+a+\frac{1}{2} a^{2}
\end{aligned}
$$

respectively. If $g \in 1+\mathfrak{N}_{3}$, then

$$
\begin{aligned}
\gamma^{m} & =\exp (m \log (\gamma)) \\
& =1+m\left((\gamma-1)+\frac{1}{2}(\gamma-1)^{2}\right)+m^{2}\left(\frac{1}{2}(\gamma-1)^{2}\right)
\end{aligned}
$$

Taking $m$ to be real extends this to a definition of the unique oneparameter subgroup in $1+\mathfrak{N}_{3}$ containing the cyclic group $\langle\gamma\rangle$. 
In particular if $\gamma_{1}, \gamma_{2} \in 1+\mathfrak{N}_{3}$ generate an action of $\mathbb{Z}^{2}$, then

$$
\begin{gathered}
\gamma_{1}^{m_{1}} \gamma_{2}^{m_{2}}=1+m_{1}\left(\left(\gamma_{1}-1\right)+\frac{1}{2}\left(\gamma_{1}-1\right)^{2}\right)+m_{2}\left(\left(\gamma_{2}-1\right)+\frac{1}{2}\left(\gamma_{2}-1\right)^{2}\right) \\
+m_{1}^{2}\left(\frac{1}{2}\left(\gamma_{1}-1\right)^{2}\right)+m_{1} m_{2}\left(\left(\gamma_{1}-1\right)\left(\gamma_{2}-1\right)\right) \\
+m_{2}^{2}\left(\frac{1}{2}\left(\gamma_{2}-1\right)^{2}\right)
\end{gathered}
$$

for $m_{1}, m_{2} \in \mathbb{Z}$. Taking $m_{1}, m_{2} \in \mathbb{R}$ defines the extension of a unipotent $\mathbb{Z}^{2}$-action to a unipotent $\mathbb{R}^{2}$-action. (This is a special case of the Mal'cev extension of a finitely generated torsionfree nilpotent group to a 1-connected nilpotent Lie group. See Mal'cev [20], or Chapter II of Raghunathan [24] for the general construction.) For the application to complete affine manifolds with virtually polycyclic fundamental group, see $\S 1$ of [7].

Non-Riemannian structures. Let $\epsilon \in \mathbb{R}$. The mappings

$$
\begin{aligned}
\mathbb{A}^{2} \stackrel{\Phi_{\epsilon}}{\longmapsto} \mathbb{A}^{2} \\
{\left[\begin{array}{l}
x \\
y
\end{array}\right] \longmapsto\left[\begin{array}{c}
x+\epsilon y^{2} / 2 \\
y
\end{array}\right] }
\end{aligned}
$$

define a one-parameter subgroup of polynomial diffeomorphisms of $\mathbb{A}^{2}$.

For $(s, t) \in \mathbb{R}^{2}$, let $T_{s, t}$ denote translation by $(s, t)$ :

$$
\begin{aligned}
& \mathbb{A}^{2} \stackrel{T_{s, t}}{\longmapsto} \mathbb{A}^{2} \\
& {\left[\begin{array}{l}
x \\
y
\end{array}\right] \longmapsto\left[\begin{array}{l}
x+s \\
y+t
\end{array}\right] .}
\end{aligned}
$$

$\Phi_{\epsilon}$ conjugates translations to the affine action $\mathbb{R}^{2} \stackrel{\rho_{\epsilon}}{\longrightarrow} \operatorname{Aff}(2)$ defined by:

$$
\rho_{\epsilon}\left(\left[\begin{array}{l}
s \\
t
\end{array}\right]\right):\left[\begin{array}{l}
x \\
y
\end{array}\right] \stackrel{\Phi_{\epsilon} T_{s, t} \Phi_{-\epsilon}}{\longrightarrow}\left[\begin{array}{c}
x+\epsilon y t+\left(s+\epsilon t^{2} / 2\right) \\
y+t
\end{array}\right] .
$$

In the standard linear representation (1.1), this affine representation equals:

$$
\rho_{\epsilon}\left(\left[\begin{array}{l}
s \\
t
\end{array}\right]\right)=\left[\begin{array}{ccc}
1 & \epsilon t & s+\epsilon t^{2} / 2 \\
0 & 1 & t \\
0 & 0 & 1
\end{array}\right]
$$


Automorphisms. The polynomial one-parameter group $\Phi_{\epsilon}$ on $\mathbb{A}^{2}$ interacts with three linear one-parameter groups on on $\mathbb{A}^{2}$ :

- Dilations defined by

$$
\left[\begin{array}{l}
x \\
y
\end{array}\right] \stackrel{\delta_{\lambda}}{\longmapsto}\left[\begin{array}{c}
\lambda^{2} x \\
\lambda y
\end{array}\right]
$$

for $\lambda>0$;

- Shears defined by

$$
\left[\begin{array}{l}
x \\
y
\end{array}\right] \stackrel{\sigma_{u}}{\longmapsto}\left[\begin{array}{c}
x+u y \\
y
\end{array}\right]
$$

for $u \in \mathbb{R}$;

- Homotheties defined by

$$
\left[\begin{array}{l}
x \\
y
\end{array}\right] \stackrel{h_{\lambda}}{\longmapsto}\left[\begin{array}{l}
\lambda x \\
\lambda y
\end{array}\right]
$$

for $\lambda \neq 0$.

Dilations and shears commute with $\Phi_{\epsilon}$, while homotheties conjugate the various elements of the one-parameter group $\Phi$ :

$$
\left(h_{\lambda}\right)^{-1} \Phi_{\epsilon} h_{\lambda}=\Phi_{\lambda \epsilon} .
$$

(3.7) implies that if $\epsilon \neq 0$, then the group $\rho_{\epsilon}\left(\mathbb{R}^{2}\right)$ is affinely conjugate to $\rho_{1}\left(\mathbb{R}^{2}\right)$.

When $\epsilon \neq 0$, different lattices in $\mathbb{R}^{2}$ give inequivalent affine structures (unlike the Euclidean case $\epsilon=0$ ). The map $\Phi_{\epsilon}$ is a polynomial developing map for this structure.

Every complete affine structure on $T^{2}$ arises from this construction.

Denote the group of orientation-preserving linear automorphisms of $\mathbb{R}^{2}$ by $\mathrm{GL}_{+}(2, \mathbb{R})$. Given

$$
R=\left[\begin{array}{ll}
s_{1} & s_{2} \\
t_{1} & t_{2}
\end{array}\right] \in \mathrm{GL}(2, \mathbb{R})
$$

and $\epsilon \in \mathbb{R}$, we define a marked affine manifold $(f, M)$ as follows. Represent $S=T^{2}$ as $\mathbb{A}^{2} / \pi$, where $\pi \cong \mathbb{Z}^{2}$ acts by integer translations. Then $\Phi_{\epsilon}$ defines a developing map

$$
\tilde{S}=\mathbb{R}^{2} \stackrel{\tilde{f}=\Phi_{\epsilon} \circ R}{\longrightarrow} \mathbb{A}^{2}
$$

and a holonomy homomorphism $h=h_{(\epsilon, R)}$

$$
\begin{aligned}
\pi & \stackrel{h}{\longrightarrow} \operatorname{Aff}(2) \\
{\left[\begin{array}{c}
m_{1} \\
m_{2}
\end{array}\right] } & \longmapsto \rho_{\epsilon}\left(\left[\begin{array}{c}
m_{1} s_{1}+m_{2} s_{2} \\
m_{1} t_{1}+m_{2} t_{2}
\end{array}\right]\right) .
\end{aligned}
$$


The corresponding affine manifold $M$ is the quotient $\mathbb{A}^{2} / h(\pi)$ and the marking $f$ is the diffeomorphism induced by $\tilde{f}$ above.

The Mal'cev construction (3.1) extends every proper affine action of $\mathbb{Z}^{2}$ on $\mathbb{A}^{2}$ to a simply transitive affine action of $\mathbb{R}^{2}$ on $\mathbb{A}^{2}$. By Kuiper [18], Furness-Arrowsmith [9], Nagano-Yagi [23], Fried-Goldman [7], this subgroup is conjugate to the subgroup

$$
G_{1}:=\rho_{1}\left(\mathbb{R}^{2}\right)=\left\{\left[\begin{array}{ccc}
1 & t & s+t^{2} / 2 \\
0 & 1 & t \\
0 & 0 & 1
\end{array}\right] \mid s, t \in \mathbb{R}\right\} .
$$

The affine representations $\rho_{\epsilon}$ define simply transitive affine actions and the evaluation map

$$
G=\mathbb{R}^{2} \longrightarrow \mathbb{A}^{2}
$$

is the developing map for an invariant affine structure on the abelian Lie group $G$.

The automorphisms of the affine structure of $G$ which normalize the multiplications in $G$ are induced by the normalizer $\operatorname{Norm}_{+}\left(G_{1}\right)$ of the image of $G$ in $\operatorname{Aff}(2)$. This normalizer is generated by two commuting one-parameter subgroups $\delta_{\lambda}$ of dilations and shears $\sigma_{u}$ defined in (3.4) and (3.5) respectively.

When $\epsilon=0$, the corresponding normalizer is larger: in this case the normalizer of the translation group is the full affine group. Every automorphism of the vector group $\mathbb{R}^{2}$ is an element of $\mathrm{GL}(2, \mathbb{R})$.

It follows that the two pairs $\left(\epsilon_{i}, R_{i}\right)$ (where $\left.i=1,2\right)$ determine the same point in $\mathfrak{D}_{c}\left(T^{2}\right)$ if and only if:

- $\epsilon_{1}=\epsilon_{2}=0$; or

- $\epsilon_{i} \neq 0$ for $i=1,2$ and

$$
R_{2}=\left(\frac{\epsilon_{1}}{\epsilon_{2}}\right)^{2} \delta_{\lambda} \sigma_{u} R_{1}
$$

for some $\lambda>0$ and $u \in \mathbb{R}$.

Polynomial tensors. An oriented non-Riemannian complete affine 2-torus $M$ admits a unique parallel area form $\omega_{M}$ of area 1 , as well as a unique parallel 1-form $\eta_{M}$ satisfying the conditions of the following Lemma. We call $\eta_{M}$ the canonical parallel 1-form on $M$.

Lemma 3.1. Let $M$ be a 2-torus with a non-Riemannian complete affine structure and parallel 2-form $\omega_{M}$ such that

$$
\int_{M} \omega_{M}=1
$$


Then there exists a unique parallel vector field $\zeta_{M}$ such that

$$
\zeta_{M}=\nabla_{\xi_{M}} \xi_{M}
$$

for a polynomial vector field $\xi_{M}$ and

$$
\omega_{M}\left(\zeta_{M}, \xi_{M}\right)=1 \text {. }
$$

There is a unique parallel 1-form $\eta_{M}$ such that

$$
\eta_{M}=\iota_{\zeta_{M}}\left(\omega_{M}\right)
$$

where $\iota$ denotes interior multiplication. Furthermore $\xi_{M}$ is unique up to addition of a constant multiple of $\zeta_{M}$.

Proof. Choose coordinates so that $M=\mathbb{A}^{2} / \Gamma$ where $\Gamma \subset G_{1}$ is a lattice. Dilations distort area by

$$
\left(\delta_{\lambda}\right)^{*} d x \wedge d y=\lambda^{3} d x \wedge d y
$$

so replacing $\Gamma$ by $\delta_{\lambda} \Gamma \delta_{\lambda}^{-1}$, we may assume that

$$
\omega=d x \wedge d y
$$

induces an area form on $M$ with area 1 .

The $\Gamma$-invariant polynomial vector fields on $\mathbb{A}^{2}$ are $G_{1}$-invariant, and

$$
\xi:=\frac{\partial}{\partial y}+x \frac{\partial}{\partial y}, \quad \zeta:=\frac{\partial}{\partial x}
$$

is a basis for the $\Gamma$-invariant polynomial vector fields on $\mathbb{A}^{2}$. Every parallel vector field is a constant multiple of $\zeta$. Furthermore $\xi, \zeta, \omega$ satisfy:

$$
\nabla_{\xi} \xi=\zeta, \quad \omega(\zeta, \xi)=1 .
$$

Let $\xi_{M}, \zeta_{M}$ be the vector fields on $M$ induced by $\xi, \zeta$ respectively. This establishes existence.

Every polynomial vector field is of the form

$$
\xi^{\prime}=a \xi+b \zeta
$$

and determines a parallel vector field

$$
\zeta^{\prime}=\nabla_{\xi^{\prime}} \xi^{\prime}=a^{2} \zeta
$$

which satisfies

$$
\omega\left(\zeta^{\prime}, \xi^{\prime}\right)=a^{3}
$$

Thus the coefficient in (3.15) is $a=1$ and conditions (3.14) uniquely determine $\zeta^{\prime}=\zeta$. Finally (3.13) uniquely determines $\eta_{M}$. 


\section{Periods as parameters}

Let $(f, M)$ be a marked complete affine 2-torus such that $M$ is nonRiemannian. Let $\eta_{M}$ be its canonical parallel 1-form. Let $\alpha_{1}, \alpha_{2} \in \pi$ be the standard basis for $\pi \cong \mathbb{Z}^{2}$. The pair

$$
\mathrm{p}:=\mathrm{p}(f, M):=\left(t_{1}, t_{2}\right) \in \mathbb{R}^{2}
$$

where

$$
t_{i}=\int_{\alpha_{i}} f^{*} \eta_{M}
$$

for $i=1,2$, is an invariant of $(f, M)$. Call $\mathrm{p}(f, M)$ the period class of $(f, M)$. If $M$ is Euclidean, define $\mathrm{p}(f, M)$ to equal $(0,0)$.

Lemma 4.1. If $M$ is non-Riemannian, the period class $\mathrm{p}(f, M)$ is nonzero.

Proof. Suppose that $\mathrm{p}(f, M)=0$. Since the images of $\alpha_{1}, \alpha_{2}$ generate $H_{1}(M)$, the cohomology class $\left[\omega_{M}\right] \in H^{1}(M ; \mathbb{R})$ is zero and $\omega_{M}$ is exact. Thus $\omega_{M}=d \psi$ for a smooth function $M \stackrel{\psi}{\rightarrow} \mathbb{R}$. Since $\omega_{M}$ is nowhere vanishing, $\psi$ is a submersion, contradicting compactness of $M$.

Since the value of the period class depends only on the cohomology class of $f^{*} \eta_{M}$, the map $\mathrm{p}:[f, M] \mapsto \mathrm{p}(f, M)$ is well defined on isotopy classes of marked tori.

Theorem 4.2. The period mapping

$$
\mathfrak{D}_{c}\left(T^{2}\right) \stackrel{\mathrm{p}}{\rightarrow} \mathbb{R}^{2}
$$

is a homeomorphism.

Proof. Suppose that $(f, M)$ is a non-Riemannian marked torus, defined by parameters $(\epsilon, R) \in \mathbb{R} \times \mathrm{GL}_{+}(2, \mathbb{R}), \epsilon \neq 0$, as in (3.8). Since $\Phi_{\epsilon}$ is area-preserving $d x \wedge d y$ induces an area form on $M$ which has total area $A=s_{1} t_{2}-s_{2} t_{1}$. Therefore $\omega_{M}$ is the area form on $M$ induced by

$$
\omega=A^{-1} d x \wedge d y .
$$

A basis for the $G_{\epsilon}=\rho_{\epsilon}\left(\mathbb{R}^{2}\right)$-invariant polynomial vector fields on $\mathbb{A}^{2}$ is

$$
\frac{\partial}{\partial y}+\epsilon y \frac{\partial}{\partial x}, \frac{\partial}{\partial x}
$$

and we compute $c \in \mathbb{R}$ for which

$$
\xi:=c\left(\frac{\partial}{\partial y}+\epsilon y \frac{\partial}{\partial x}\right)
$$


induces polynomial vector fields $\xi_{M}, \zeta_{M}$ satisfying (3.14). Define

$$
\zeta=\nabla_{\xi} \xi=c^{2} \epsilon \frac{\partial}{\partial x}
$$

so that $\omega(\xi, \zeta)=A^{-1} c^{3} \epsilon$. Thus (3.12) implies

$$
c=\left(\frac{A}{\epsilon}\right)^{1 / 3}
$$

so the canonical vector field, and canonical parallel 1-form on $M$ are represented by

$$
\begin{aligned}
\zeta & =A^{2 / 3} \epsilon^{1 / 3} \frac{\partial}{\partial x} \\
\eta=\iota_{\zeta} \omega & =\left(\frac{\epsilon}{A}\right)^{1 / 3} d y
\end{aligned}
$$

respectively. The period class for $[f, M]$ is:

$$
\mathrm{p}=\left(\frac{\epsilon}{A}\right)^{1 / 3}\left[\begin{array}{l}
t_{1} \\
t_{2}
\end{array}\right]
$$

$\mathrm{p}$ is surjective: We construct an inverse $\mathrm{q}$ to $\mathrm{p}$ as follows. Let $\mathrm{k}=$ $\left(k_{1}, k_{2}\right) \in \mathbb{R}^{2}$. Define

$$
R_{\mathrm{k}}:=\|\mathrm{k}\|^{2}\left[\begin{array}{cc}
k_{2} & -k_{1} \\
k_{1} & k_{2}
\end{array}\right]
$$

where

$$
\|\mathrm{k}\|=\sqrt{\left(k_{1}\right)^{2}+\left(k_{2}\right)^{2}}
$$

as usual. Then

$$
\Psi_{\mathrm{k}}:=\left(R_{\mathrm{k}}\right)^{-1} \circ \Phi_{1} \circ R_{\mathrm{k}}=\left(Q_{\mathrm{k}}\right)^{-1} \circ \Phi_{\|\mathrm{k}\|^{3}} \circ Q_{\mathrm{k}}
$$

is a polynomial developing map, where $Q_{\mathrm{k}}$ is the orthogonal matrix

$$
Q_{\mathrm{k}}:=\|\mathrm{k}\|^{-3} R_{\mathrm{k}}=\left[\begin{array}{cc}
k_{2} /\|\mathrm{k}\| & -k_{1} /\|\mathrm{k}\| \\
k_{1} /\|\mathrm{k}\| & k_{2} /\|\mathrm{k}\|
\end{array}\right]
$$

when $\mathrm{k} \neq 0$. (Compare (3.7).) Explicitly,

$$
\left[\begin{array}{l}
x \\
y
\end{array}\right] \stackrel{\Psi_{\mathrm{k}}}{\longrightarrow}\left[\begin{array}{l}
x \\
y
\end{array}\right]+\frac{1}{2}\left(k_{1} x+k_{2} y\right)^{2}\left[\begin{array}{c}
k_{2} \\
-k_{1}
\end{array}\right]
$$

so $\Psi_{\mathrm{k}}$ depends smoothly on $\mathrm{k} \in \mathbb{R}^{2}$. Thus in particular $\mathrm{k} \mapsto \Psi_{\mathrm{k}}$ defines a map

$$
\mathbb{R}^{2} \stackrel{\mathrm{q}}{\rightarrow} \mathfrak{D}_{c}\left(T^{2}\right)
$$

which is continuous. 
Note also that $\Psi_{\mathrm{k}}^{-1}=\Psi_{-\mathrm{k}}$. Hence the corresponding holonomy representation

$$
h_{\mathrm{k}}=\Psi_{\mathrm{k}} T \Psi_{-\mathrm{k}}
$$

depends smoothly on k. Furthermore $h_{\mathrm{k}}$ equals the conjugate of $h_{(\epsilon, R)}$ by $R^{-1}$, where

$$
\epsilon=\|\mathrm{k}\|^{3}, R=Q_{\mathrm{k}} .
$$

By (4.1), the period class of $\mathrm{q}(\mathrm{k})$ equals $\mathrm{k}$. Thus the composition

$$
\mathbb{R}^{2} \stackrel{\mathrm{q}}{\rightarrow} \mathfrak{D}_{c}\left(T^{2}\right) \stackrel{\mathrm{p}}{\rightarrow} \mathbb{R}^{2}
$$

is the identity map on $\mathbb{R}^{2}$, and $\mathrm{p}$ is surjective.

Note also that $\mathrm{q}$ is surjective. Indeed, since every element $R \in$ $G L_{+}(2, \mathbb{R})$ decomposes as a product of an orthogonal and an uppertriangular matrix, (3.10) implies that every element of $\mathfrak{D}_{c}\left(T^{2}\right)$ may be represented by a holonomy representation with parameters $(\epsilon, Q)$, where $Q \in S O(2)$ is an orthogonal matrix. Therefore, (4.5) shows that $\mathrm{q}$ is a continuous bijection onto $\mathfrak{D}_{c}\left(T^{2}\right)$.

$\mathrm{p}$ is continuous: It follows from (4.3) that the set $S=\left\{\Psi_{\mathrm{k}} \mid \mathrm{k} \in \mathbb{R}^{2}\right\}$ is closed in the space of development maps $\operatorname{Def}_{c}\left(T^{2}\right)$, and hence $\mathfrak{D}_{c}\left(T^{2}\right)$ carries the quotient topology from $\mathrm{S}$. Since $\mathrm{q}$ is a bijection, $\mathrm{S}$ is actually homeomorphic to $\mathfrak{D}_{c}\left(T^{2}\right)$. Write $\Psi_{\mathrm{k}}=\left(\Psi_{\mathrm{k}}^{1}, \Psi_{\mathrm{k}}^{2}\right)$. Since

$$
k_{1}=-\sqrt[3]{\frac{\partial^{2}}{\partial y^{2}} \Psi_{\mathrm{k}}^{2}} \text { and } k_{2}=\sqrt[3]{\frac{\partial^{2}}{\partial x^{2}} \Psi_{\mathrm{k}}^{1}},
$$

$\mathrm{p}: \mathrm{S} \rightarrow \mathbb{R}^{2}$ is continuous. Therefore, $\mathrm{p}$ is a continuous function on the deformation space as well.

$\mathrm{p}$ is injective: Let $[(f, M)]$ be a point in in $\mathfrak{D}_{c}\left(T^{2}\right)$. If $\mathrm{p}(f, M)=0$, then by Lemma 4.1, $M$ must be Euclidean. All Euclidean structures correspond to a single point in $\mathfrak{D}_{c}\left(T^{2}\right)$.

Now suppose that $M$ is non-Riemannian. Since $\mathrm{q}$ is a bijection onto $\mathfrak{D}_{c}\left(T^{2}\right)$, the composition (4.5) implies that $\mathrm{p}$ is injective on nonRiemannian structures as well.

It follows that $\mathrm{p}$ is a homeomorphism with inverse $\mathrm{q}$.

The moduli space. The moduli space of complete affine structures on $T^{2}$ is obtained as the quotient space of $\mathfrak{D}_{c}\left(T^{2}\right)$ by the mapping class group $\pi_{0}\left(\operatorname{Diff}\left(T^{2}\right)\right)$.

Explicitly, the action of the modular group $\mathrm{SL}(2, \mathbb{Z})$ on $\mathfrak{D}_{c}\left(T^{2}\right)=\mathbb{R}^{2}$ is given as follows. Let $(f, M)$ be an affine manifold marked by $S=T^{2}$, 
and $\phi_{A}$ the diffeomorphism of $T^{2}$ which corresponds to $A \in \mathrm{SL}(2, \mathbb{Z})$. Then the action of $A$ on $\mathfrak{D}_{c}\left(T^{2}\right)$ is represented by $(f, M) \mapsto\left(f \circ \phi_{A}, M\right)$.

\section{Lemma 4.3.}

$$
\mathrm{p}\left(f \circ \phi_{A}, M\right)=A^{t}(\mathrm{p}(f, M))
$$

Proof. We assume that $\tilde{f}: \mathbb{A}^{2} \rightarrow \mathbb{A}^{2}$ is of the form $\Phi_{\epsilon} \circ R$, for some linear map $R \in \mathrm{GL}(2, \mathbb{R})$ with determinant 1 . By $(4.1), \mathrm{p}(f, M)=\epsilon^{\frac{1}{3}}\left(t_{1}, t_{2}\right)^{t}$, where $\left(t_{1}, t_{2}\right)$ is the second row vector of $R$. Then

$$
\widetilde{f \circ \phi_{A}}=\tilde{f} \circ A=\Phi_{\epsilon} \circ R A
$$

and $\mathrm{p}\left(f \circ \phi_{A}, M\right)=\epsilon^{\frac{1}{3}} A^{t}\left(t_{1}, t_{2}\right)^{t}=A^{t}(\mathrm{p}(f, M))$.

Thus the moduli space identifies naturally with the quotient of $\mathbb{R}^{2}$ by $\operatorname{SL}(2, \mathbb{Z})$.

Differentiable families. We construct a differentiable family of complete affine tori over the deformation space: For $\mathrm{k} \in \mathbb{R}^{2}$, we let $h_{\mathrm{k}}$ : $\mathbb{Z}^{2} \rightarrow \operatorname{Aff}(2)$ denote the holonomy homomorphism for the developing map $\Psi_{\mathrm{k}}$ (see proof of Theorem 4.2). We let $\mathbb{Z}^{2}$ act on $\mathbb{R}^{2} \times \mathbb{A}^{2}$ via

$$
\gamma:(\mathrm{k}, v) \mapsto\left(\mathrm{k}, h_{\mathrm{k}}(\gamma) v\right)
$$

By (4.4), $\mathbb{Z}^{2}$ acts differentiably (in fact by polynomial diffeomorphisms), and the quotient space $\mathcal{F}$ is a differentiable manifold, and a torusbundle over $\mathbb{R}^{2}$ via the projection onto the first factor. This bundle is trivial as a differentiable torus bundle. The diffeomorphism $(\mathrm{k}, v) \mapsto\left(\mathrm{k}, \Psi_{\mathrm{k}}(v)\right)$ induces an explicit trivialization

$$
\mathbb{R}^{2} \times T^{2} \rightarrow \mathcal{F}
$$

The bundle $\mathcal{F} \rightarrow \mathbb{R}^{2}$ admits a system of locally trivializing charts in $\mathbb{R}^{2} \times \mathbb{A}^{2}$ which define an affine structure on the fibers, giving a differentiable family of affine manifolds over $\mathbb{R}^{2}$ (see [17] for the definition of differentiable family of complex manifolds). Each fibre of the corresponding differentiable family

$$
\mathcal{F} \longrightarrow \mathfrak{D}_{c}\left(T^{2}\right)
$$

is a marked complete affine torus which represents its base-point in the deformation space.

Not every differentiable family of complete affine tori is induced by a smooth map into the deformation space. Indeed, for $a \in \mathbb{R}$, let us consider the development map $\Phi_{a}: \mathbb{R}^{2} \rightarrow \mathbb{A}^{2}$ defined by

$$
\left[\begin{array}{l}
x \\
y
\end{array}\right] \stackrel{\Phi_{a}}{\longrightarrow}\left[\begin{array}{l}
x \\
y
\end{array}\right]+\frac{1}{2} x^{2}\left[\begin{array}{l}
0 \\
a
\end{array}\right] \text {. }
$$


Then $\Phi_{a}, a \in \mathbb{R}$, constitutes a differentiable family of developing maps, and there is a corresponding differentiable family of affine manifolds

$$
\mathcal{E} \longrightarrow \mathbb{R}
$$

where each fibre $\mathcal{E}_{a}, a \in \mathbb{R}$, is a complete affine torus marked by $\Phi_{a}$. (Since the parameter $a$ enters linearly, the family $\mathcal{E} \rightarrow \mathbb{R}$ defines an affine fibration, see [31].) The corresponding curve $\mathbb{R} \rightarrow \mathfrak{D}_{c}\left(T^{2}\right)$ is a line in $\mathbb{R}^{2}$, parametrized by

$$
a \mapsto \mathrm{p}\left(\mathcal{E}_{a}\right)=\left(0,-a^{1 / 3}\right) .
$$

It is not smooth in $a=0$.

As mentioned in the introduction, such a phenomenon can not happen for deformations of elliptic curves and their induced maps into $\mathrm{H}^{2}$, see [17, Theorem 14.3].

\section{REFERENCES}

[1] O. Baues, Gluing affine two-manifolds with polygons, Geom. Dedicata 75 (1999), no. 1, 33-56.

[2] , Varieties of discontinuous groups, in Crystallographic groups and their generalizations, Contemporary Mathematics 262 (2000), 147-158.

[3] , Finite extensions and unipotent shadows of affine crystallographic groups, C. R. Math. Acad. Sci. Paris 335 (2002), no. 10, 785-788.

[4] Y. Benoist, Tores affines, in "Crystallographic groups and their generalizations" (Kortrijk, 1999), 1-37, Contemp. Math., American Mathematical Society (2000).

[5] J.P. Benzecri, Variétés localement affines, Séminaire Ehresmann, 1959.

[6] R.D. Canary, D.B.A. Epstein, P. Green, Notes on notes of Thurston, Analytical and Geometric Aspects of Hyperbolic Space, London Mathematical Society Lecture Note Series 111, Cambridge University Press, (1984).

[7] D. S. Fried, W.M. Goldman, Three-dimensional affine crystallographic groups, Adv. in Math. 47 (1983), no. 1, 1-49.

[8] D. Fried, W.M. Goldman, M. W. Hirsch, Affine manifolds with nilpotent holonomy, Comm. Math. Helv. 56 (1981), 487-523.

[9] P. Furness, D. Arrowsmith, Locally symmetric spaces, J. London Math. Soc. (2) 10 (1972), 487-499.

[10] W.M. Goldman, Affine manifolds and projective geometry on surfaces, Senior thesis, Princeton University (1977).

[11] _ Projective structures with Fuchsian holonomy, J. Diff. Geom. 25 (1987), 297-326.

[12] _ Projective geometry on manifolds, Lecture notes v.0.3 (1988).

[13] _ Geometric structures on manifolds and varieties of representations, Contemporary Mathematics 74 (1988), 169-198.

[14] $\quad$ On the polynomial cohomology of affine manifolds, Invent. Math. 65 $(1981 / 82)$, no. $3,453-457$.

[15] M. Kapovich, "Hyperbolic manifolds and discrete groups", Progress in Mathematics, 183 Birkhäuser Boston, Inc. (2001). 
[16] S. Kobayashi, K. Nomizu, "Foundations of Differential Geometry, I", John Wiley \& Sons (1969).

[17] K. Kodaira, D.C. Spencer, On deformations of complex analytic structures I, Ann. of Math. (2) 67 (1958), 328-466.

[18] N.H. Kuiper, Sur les surfaces localement affines, Géométrie différentielle. Colloques Internationaux du Centre National de la Recherche Scientifique, Strasbourg 1953, 79-87.

[19] W. L. Lok, Deformations of locally homogeneous spaces and Kleinian groups, Doctoral dissertation, Columbia University 1984.

[20] A. I. Mal'cev, On a class of homogeneous spaces, Amer. Math. Soc. Transl. 39 (1951).

[21] Y. Matsushima, Affine structures on complex manifolds, Osaka J. Math. 5 (1968), 215-222.

[22] J. Milnor, On the existence of a connection with curvature zero, Comment. Math. Helv. 32 (1958), 215-223.

[23] T. Nagano, K. Yagi, The affine structures on the real two-torus, Osaka J. Math. 11 (1974), 181-210.

[24] M. Raghunathan, "Discrete Subgroups of Lie Groups," Springer-Verlag (1972).

[25] S. Schwartzman, Asymptotic cycles, Ann. Math. 66 (2), (1957) 270-284.

[26] J. Smillie, Affinely flat manifolds, Doctoral Thesis, University of Chicago (1977).

[27] J. Stillwell, "Classical Topology and Combinatorial Group Theory," Graduate Texts in Mathematics 72, Springer Verlag (1993).

[28] D. Sullivan and W. Thurston, Manifolds with canonical coordinate charts: some examples, L'Enseignement Math. 29 (1983), 15-25.

[29] W. Thurston, The Geometry and Topology of 3-Manifolds, unpublished lecture notes, Princeton University (1979).

[30] _ Three dimensional geometry and topology, Vol.1, Princeton University Press (1997).

[31] A. Tsemo, Fibrés affines, Michigan Math. J. 49 (2001), no. 3, 459-484.

[32] J.A. Wolf, Spaces of constant curvature, Publish or Perish (1984).

Departement Mathematik, ETH-Zentrum, Rämistrasse 101, CH-8092

ZÜRICH, SWITZERLAND

E-mail address: oliver@math.ethz.ch

Mathematics Department, University of Maryland, College Park, MD 20742 USA

E-mail address: wmg@math.umd.edu 